\title{
Adoption du palmier à huile en Côte d'Ivoire
}

\section{Adoption of oil palm in Côte d'Ivoire}

Oléagineux, Corps Gras, Lipides. Volume 7, Numéro 2, 155-65, Mars - Avril 2000, Dossier : Afrique, plantation et développement

Auteur(s) : Serge NAÏ NAÏ, Emmanuelle CHEYNS, François RUF

Résumé : Au cours des années 70 et 80 , et en dépit de problèmes fonciers, l'effort de l'État ivoirien pour promouvoir les cultures du palmier et de l'hévéa à travers des projets et des sociétés d'encadrement spécialisées a connu un succès certain [1-5]. Un des intérêts de ces formes d'investissements a été d'apporter du crédit et de l'information aux planteurs, notamment sous forme de matériel végétal, d'engrais et d'encadrement technique. Dans le cas de la filière palmier, information, capital et disponibilité en matériel végétal ont été des facteurs essentiels à l'adoption de cette « nouvelle » culture pérenne.

Summary: Two government-sponsored programmes in 1963-1984 and 1985-1990 played a major role for promoting oil palm as a diversification crop in Côte d'Ivoire, the agriculture of which is mostly based on cocoa and coffee. The main role of these projects was to bring information, planting material and capital through credit schemes. Part of these resources had been captured by government and extension staff who set up large oil palm farms for themselves, usually at the expense of forest reserves. Nevertheless, thousands of farmer families also benefited information and credit schemes from these programmes. Then in the 1990s, government programmes were swept by liberalisation winds. It was feared that without information and capital brought by projects, the rate of oil palm adoption would sink. Surveys show the opposite trend. Since 1995, the rush to selected seeds and oil palm investments is still stronger than during the era of programmes providing seeds on a credit base. Many farmers could find a way to overcome capital constraints. Another factor of adoption is the exhaustion of the forest rent and replanting difficulties faced by cocoa farmers. It is easier to replant oil palm after ageing cocoa and coffee farms. Regular and monthly revenues of oil palm also prove to be quite attractive compare to cocoa. The drop of cocoa and coffee prices from 1988 to 1993 while that of oil palm was relatively sustained also encouraged the process. Eventually, the opportunity to get a return from palm wine (widely consumed in WestAfrica) after cutting down the palm tree is also aknowledged by farmers as a strong incentive to adopt the tree crop. Oil palm looks like an ideal saving tool and patrimony, ready to be used when farmers face an urgent need of cash.

Keywords: oil palm, tree crop, diversification, replanting, credit, forest rent, selected seed, comparative prices, palm wine, Côte d'Ivoire.

\section{ARTICLE}

\section{Phases d'adoption du palmier à huile : des « plans » de développement à la privatisation}

De 1963 à 1990, 143000 ha de palmier à huile ont été plantés. Deux " plans palmier » ont largement contribué à ce résultat, par l'injection de financements importants. L'accroissement des superficies 
villageoises s'est surtout réalisé lors du deuxième plan, entre 1985 et 1990. Depuis la privatisation en 1997, tous les financements de plantations villageoises à crédit sont supprimés, pouvant laisser craindre un relatif abandon de la culture. Pourtant, il s'est produit le phénomène opposé. Sur les cinq dernières années, de 1995 à $2000^{4}$, l'accroissement des superficies villageoises plantées a été encore plus marqué que lors du deuxième plan palmier.

\section{De 1963 à 1990 : les plans palmier}

Dans un objectif de diversification des activités agricoles en zones forestières, le Gouvernement ivoirien et la Communauté économique européenne signent, en 1963, une convention de financement qui prévoit l'octroie d'une aide non remboursable de 1,15 milliard de FCFA. Son objet est de promouvoir la culture du palmier à huile sélectionné dans les milieux villageois de la partie sud-est du pays. Elle constitue la première phase d'un plan de développement de la production d'huile de palme, le " premier plan palmier ", auquel succédera un deuxième plan à partir de 1985 . De 1963 à 1990 vont se succéder une série de financements destinés à promouvoir le palmier sélectionné dans un cadre agro-industriel comprenant des usines de grandes capacités (à partir de 20 $\mathrm{t} / \mathrm{h}$ ), des blocs de plantations industrielles (PI), des plantations villageoises (PV) et, à partir de 1985, des plantations dites PMEA (petites et moyennes entreprises agricoles). En 1964 est institué un « Fonds d'extension et de renouvellement " de la culture du palmier à huile (le FER) destiné au crédit à court, moyen et long termes des réalisations agricoles. Les planteurs villageois disposent ainsi d'un financement de leurs plantations à crédit, d'abord remboursable de façon individuelle par annuité fixe, puis prélevé sous forme de cotisation par kilo de régime au moment de la vente. Pour mener à bien ce développement, l'État ivoirien crée la Société pour le développement de l'exploitation du palmier à huile (Sodepalm) puis, en 1969, deux sociétés d'économie mixte, Palmindustrie et Palmivoire. Ces trois sociétés sont propriétaires des plantations et installations de traitement industriel, et chargées de la gestion des ensembles agro-industriels. Ces structures juridiques établies, une série de financements est concédée, à 32 milliards de FCFA au cours des cinq années suivantes, dans le cadre du premier plan palmier [9] (tableau 1). À partir de 1975, une réforme des structures juridiques entraîne de graves difficultés financières et, à partir de 1979, un arrêt des plantations de palmier. Après une période de restructuration et la mise en œuvre d'un programme intérimaire de relance, un deuxième plan palmier visant à redynamiser le développement de la culture du palmier à huile est conclu. II porte sur un financement de 90 milliards de FCFA (dont 33 milliards en apports externes) et s'étend de 1985 à 1990 (tableau 1).

\section{Phases de croissance du palmier sélectionné}

Les données disponibles sur les superficies plantées depuis 1963 laissent apparaître trois phases de forte croissance, les deux premières correspondent aux années financées, la troisième à la privatisation de la filière.

\section{Années financées}

Le premier plan palmier a permis une croissance régulière des superficies plantées cumulées jusqu'en 1978 (figures 1 et 2), avec notamment des pics de croissance dans les années 1968-1970 puis 1976-1978. Cette période correspond essentiellement à une croissance des superficies des blocs industriels et l'installation d'usines ${ }^{5}$. La fin du premier plan palmier est marquée par un arrêt des superficies plantées entre 1979 et 1982, du fait de graves problèmes de gestion financière, dont les 
effets sur la production pourraient être apparents dans les années 2005.

Le programme intérimaire et le deuxième plan palmier (90 milliards de FCFA) marquent un nouvel élan dans l'accroissement des superficies plantées, notable dans les années 1983 à 1990, avec un pic de superficies plantées en 1986 et 1987. Cette deuxième phase de développement indique, par rapport à la première, une inversion entre les proportions de superficies industrielles et villageoises ${ }^{6}$. À l'issue du premier plan palmier (en 1982), 63 \% des superficies plantées sont industrielles alors que, à l'issue du deuxième plan (en 1990), 54 \% sont des superficies villageoises. De 1963 à 1990, 72 $\%$ des plantations industrielles ont été plantées lors du premier plan palmier, alors que $65 \%$ des plantations villageoises ont été plantées lors du deuxième plan palmier (+ programme intérimaire tableau 2 et figure 1).

L'accroissement des plantations villageoises lors de cette deuxième phase est directement lié à l'offre plus importante de crédits en direction des planteurs paysans ${ }^{7}$. Les financements du premier plan palmier ont d'abord été dirigés vers la création de complexes agro-industriels et ceux du deuxième plan palmier vers le financement à crédit de nombreuses exploitations familiales (fournitures de semences et d'engrais, etc.). En outre, cet apport de crédit a très précisément coïncidé avec la diminution de la rente forestière et les difficultés de replantation cacaoyères et caféières $[8,10,11]$.

\section{5 : accès aux semences et nouvel engouement pour le palmier}

La troisième phase marque un deuxième boom dans l'accroissement des superficies villageoises; elle correspond à la période de privatisation de la filière (figures 3 et 4). À partir de 1995-1996, les planteurs ont un accès direct aux semences sélectionnées à la station de La Mé. En dehors de tout système de financement à crédit intra-filière (supprimé depuis la privatisation), environ 70000 ha de palmiers sélectionnés auraient été plantés en parcelles villageoises depuis 1995 (estimations à partir de la vente de graines germées à La Mé, qui incluent les prévisions de vente pour 2000) ${ }^{8}$. La superficie moyenne plantée en secteur villageois pendant cette période (1995-2000) est même supérieure à celle plantée lors du deuxième plan palmier (tableau 3).

En 1999, après un inventaire réalisé par les repreneurs, les superficies villageoises ont été estimées à 132000 hectares, soit 54500 ha de plus qu'en $1990^{\circ}$. On arrive donc ici à un premier résultat important : la baisse de production engendrée par l'arrêt des plantations entre 1979 et 1982, baisse théoriquement prévisible vers 2005, pourrait finalement être masquée et compensée par l'entrée en production des nouvelles plantations créées en période de libéralisation, depuis 1995.

Les raisons de l'adoption du palmier durant ces trois phases méritent une plus large analyse que nous verrons dans la troisième partie. On peut déjà souligner que l'adoption du palmier entre 1960 et 1990 est largement liée à l'offre de Palmindustrie aux planteurs, selon les choix d'allocation des ressources financières des plans de développement. La plus large adoption du palmier sélectionné en milieu villageois lors du deuxième plan est liée à ces choix politiques, mais aussi à des facteurs déterminant l'intérêt des planteurs (tels que la diminution de la rente forestière dans les années 80 ). En revanche, depuis la réorganisation de la filière à partir des années 1995, l'adoption du palmier sélectionné est presque entièrement liée à la demande des planteurs (peu de mesures incitatives ou restrictives publiques ou para-publiques et financières). 


\section{Qui sont les planteurs de palmier ?}

Dans ce contexte de forte adoption récente en dehors de tout système de crédit ou de financement, qui sont ces nouveaux planteurs et quelle est l'origine de leur capital ?

Depuis 5 ans, les planteurs ont la liberté de se rendre à la station de La Mé (CNRA) où sont produites les semences, pour les acheter. En même temps, le label «La Mé » est connu de nombreux planteurs et reconnu comme synonyme d'excellence du matériel végétal. La station devient donc un lieu privilégié pour des enquêtes sur l'adoption du palmier. La population enquêtée est celle de 80 chefs d'exploitation (ou de leurs représentants) ${ }^{10}$ qui ont acquis du matériel végétal sélectionné à La Mé. L'échantillon n'est donc pas représentatif des planteurs de palmier à huile en général : il porte sur une population de planteurs qui acquièrent directement leur matériel végétal et payent pour un matériel végétal sélectionné ${ }^{11}$. Les caractéristiques des 80 chefs d'exploitations concernés par les enquêtes sont assez particulières : $62 \%$ ont un niveau scolaire secondaire ou supérieur, $38 \%$ ont pour activité principale un métier salarié ou fonctionnaire, et $52 \%$ ne sont pas résidants sur les lieux de plantations ( $32 \%$ de l'échantillon est résidant en Abidjan).

Les cadres résidant en ville apparaissent ainsi sur-représentés par rapport à la population globale des planteurs de palmier à huile. Ils ont certainement plus de facilités pour se rendre à la station, un capital lié à leur activité salariée et, peut-être aussi, une meilleure information sur la qualité du matériel végétal. Néanmoins, tous les types de planteurs ont été touchés durant l'enquête. Par ailleurs, la courbe d'adoption du palmier tirée de l'échantillon coïncide très bien avec les données obtenues par des études antérieures, telle celle de Hirsch [6]. Dans les deux cas, on retrouve parfaitement l'accélération des plantations entre 1986 et 1988 (voir figures 5 et 6, à comparer avec les figures 3 et 4 ).

Il est de notoriété publique que les grands projets conduits par l'ancienne Palmindustrie ont donné des opportunités à de nombreux cadres ivoiriens de créer des plantations de dimension relativement importante. L'enquête de La Mé confirme sans ambiguïté un dualisme entre planteurs " cadres » et planteurs « paysans » tout en apportant des éclairages sur le contenu de chacune des catégories.

\section{Dualisme planteurs-cadres et planteurs-paysans}

À la faveur des projets, les cadres retraités et les cadres supérieurs et moyens se sont effectivement " taillé » de grandes exploitations mais les paysans qui ont commencé tôt en ont aussi profité, avec une superficie moyenne de 10 hectares (planteurs-paysans « $1 »)$ (tableau 4).

L'autre moitié de la population enquêtée est cantonnée à une moyenne de l'ordre de 3 ha. Cette moitié se partage entre des paysans exploitant moins de $50 \%$ de leur exploitation en palmier et des cadres subalternes (techniciens, instituteurs, infirmiers) en contact avec le milieu rural, habitant dans les villages. Il est probable que ces petits planteurs de palmiers sont dans un processus d'adoption récente. Comment ces planteurs se comportent-ils en 1999 au niveau de l'achat de semences et donc des projets de nouveaux investissements « palmier » en 2000 ? 
Là encore, les planteurs cadres dominent largement les investissements et cela semble montrer qu'ils restent avantagés pour l'accès au matériel végétal, après la libéralisation du secteur tout autant qu'en période de projets (tableau 5). La stratégie d'investissement des cadres retraités est particulièrement impressionnante.

Toutefois, derrière ce premier constat, on voit aussi se dessiner une accélération des investissements en palmier à huile chez les petits planteurs. D'après cette enquête, en 2000 , les deux catégories de " petits planteurs ", cadres subalternes et paysans « 2 ", vont doubler leurs moyennes de superficie de palmier à huile, de l'ordre de 3 ha à 6 ha par exploitation ${ }^{12}$. Ces chiffres évoquent donc aussi une nouvelle dynamique. La libéralisation de l'accès au matériel végétal ouvre la porte à de nouveaux candidats à la plantation de palmier, apparemment en grand nombre.

De nouveaux planteurs de palmier à la fin des années 90

Le boom sur la demande de matériel végétal signifie aussi de nouveaux planteurs de palmiers. D'après notre enquête de La Mé, 50 \% des planteurs enquêtés ont adopté le palmier pour la première fois entre 1997 et 1999 (30 \% I'ont adopté la première fois l'année de l'enquête, en $1999^{13}$ ). Le mouvement d'adoption semble avoir repris en 1995 (figure 5) et correspond en partie à un effet d'imitation : les premiers planteurs ayant obtenu un relatif succès économique, notamment ceux qui avaient planté avec les projets dans les années 1986 à 1988, la nouvelle vague démarrant à la fin des années 90 est faite de gens impressionnés par cette réussite et souhaitant y participer.

On observe également un effet démographique et de changement de génération. La moyenne d'âge des planteurs ayant adopté le palmier pour la première fois après 1997 est de 37 ans contre 45 ans pour ceux qui l'ont adopté avant 1997. Il y a bien sûr là un effet normal d'interaction entre l'âge des planteurs et l'âge des plantations. Néanmoins, on peut aussi y lire un effet " jeunes " associé à la crise économique dans le pays. Partout en Côte d'Ivoire la crise des années 90 a provoqué un reflux des jeunes scolarisés (et déscolarisés) des villes vers leurs villages. Beaucoup retrouvent de la terre et optent pour la plantation. Or la crise profonde sur les prix du cacao de 1988 à 1994 renforce les décisions d'adoption du palmier à partir de 1995, même si les prix du cacao remontent provisoirement (figure 7) et malgré la contrainte de financement sur le palmier.

Financement : contrainte sur le capital

Parmi les planteurs paysans « 2 », les principales sources de financement sont précisément le cacao et le café ou d'autres cultures ainsi qu'un complément fourni par l'aide familiale. Ceci illustre bien le processus de diversification entrepris par les planteurs de café et cacao (tableau 6).

Parmi les retraités et les paysans " 1 ", à dominante palmier, l'accumulation sur cette culture est désormais suffisante pour financer des investissements réguliers.

Enfin, parmi les cadres en activité, c'est bien le salaire qui est la principale source de financement. Ceci confirme la primauté de l'accès au capital et à un revenu extérieur dans l'adoption d'une nouvelle culture pérenne telle que le palmier sélectionné, du fait du coût d'investissement dans le matériel végétal.

Processus de déforestation ou replantation/diversification ? (tableau 7) 
L'adoption du palmier en Côte d'Ivoire participe-t-elle plutôt à un processus de déforestation ou de reformation d'une couverture végétale à partir de recolonisation de jachères ou de replantations ? Les réponses sont fort opposées selon les types de planteurs.

Parmi les planteurs paysans, ainsi que chez les instituteurs, infirmiers, et autres petits cadres locaux de l'échantillon, le palmier est indéniablement une culture venant en recolonisation des jachères, et en replantation/diversification après cacao et café. On retrouve très bien le déterminisme écologique de la diversification. À la suite des difficultés de la replantation de cacao, les planteurs optent pour le café. Si les difficultés persistent sur le café ou si des opportunités apparaissent avec des projets (et donc des financements et une prise en charge du risque de la reconversion par les sociétés industrielles), les planteurs recherchent de nouvelles solutions comme l'ananas. Ils en viennent notamment à d'autres cultures pérennes telles que le palmier ou l'hévéa [8, 10-12]. C'est d'ailleurs un phénomène universel dans l'histoire des économies cacaoyères et post-cacaoyères [3].

En revanche, parmi les cadres, le palmier à huile reste une culture entraînant directement une déforestation. Le déterminant est politique. Les cadres cherchant de grandes superficies ont pu les trouver dans les réserves de forêts. Certains projets se sont développés aux dépens des forêts dans les zones encore peu touchées par le cacao. Il s'agissait soit de réserves de forêts villageoises, soit de forêts classées. En ce cas, l'adoption du palmier, loin de correspondre à un processus de replantation/diversification coïncidant avec un épuisement de la rente forêt, a au contraire été un des agents de la déforestation. Cette forme d'adoption du palmier exprime aussi un relatif transfert des terres et des ressources publiques en capital (notamment pour couvrir les coûts du défrichement de forêt) au profit des cadres.

\section{Facteurs d'adoption du palmier à huile}

Depuis 1995, l'adoption du palmier à huile en milieu paysan est influencée par la privatisation de Palmindustrie, notamment du fait de la libéralisation de l'accès aux semences. En dehors des attraits spécifiques et structurels de cette culture, cette adoption peut s'inscrire dans des stratégies de diversification pour répondre à des problèmes d'ordre écologique et économique comme le vieillissement du verger café-cacao et la chute des cours. Mais historiquement, dans un premier temps, le grand déterminant de l'adoption du palmier sélectionné a été politique.

\section{Facteur politique et phénomène de société}

La décision de favoriser le développement du palmier revient indéniablement à une décision politique de l'État ivoirien soutenue par des bailleurs de fonds et par une Europe probablement soucieuse de diversifier ses approvisionnements en huiles alimentaires. En même temps, la décision a été rapidement relayée et démultipliée par des centaines de cadres de l'État et de ses diverses structures d'encadrement agricole. Dans ce contexte, comme le cacao, le palmier a bien été également une culture de premier cycle après défrichement de forêt, telle que dans la région d'Ehania puis ensuite de Grabo dans le Sud-Ouest. Simultanément et paradoxalement, pour les « planteurs paysans ", l'adoption du palmier a plutôt coïncidé avec la fin d'un premier cycle cacaoyer et caféier. 
Facteurs écologiques et conjonction de fin de cycles régionaux du cacao et du café

\section{Disparition de la rente forêt et vieillissement du verger café-cacao}

Le boom cacao de la Côte d'Ivoire a été largement tributaire de la rente forêt. L'installation des cacaoyères après défrichement de jachères nécessite une augmentation du temps de travail et des intrants $[8,11,13,14]$. II en résulte le mouvement de migration et de déplacements des fronts pionniers, désormais bien connu. Cependant, la raréfaction des forêts génère aussi des innovations. Au moins depuis les incendies de forêts en 1983, et plus particulièrement dans les années 90, bien des planteurs (ou plutôt leurs fils) et de nouveaux migrants reprennent des terres de jachère et innovent sur la replantation cacao et café [15]. Une autre solution au vieillissement de leurs cacaoyères ou caféières consiste à les remplacer par le palmier. Ce phénomène a été observé dans plusieurs régions. Ainsi, à l'est d'Abidjan, l'épuisement de la rente forestière a généré des opportunités pour le cocotier hybride, le palmier l'ananas, avec un succès particulier pour le palmier du fait d'un prix rémunérateur, de la régularité de la collecte et des revenus mensualisés [16]. D'après Léonard [11] à Sassandra, où la plus grande partie des superficies de palmier a été plantée après 1985 (3 371 ha sur 5200 ha plantés entre 1967 et 1990), " l'engouement pour la culture du palmier correspond pour une large part à un objectif de reconversion ou de diversification face au vieillissement des vergers caféiers et cacaoyers et aux difficultés de replantation ».

\section{Privatisation de Palmindustrie : disponibilité des semences}

L'installation de toutes les palmeraies sélectionnées était autorisée par la société d'encadrement (Sodepalm puis Palmindustrie). Le contrôle exercé par cette structure avait une influence sur le rythme d'adoption du palmier. Nous avons vu que ce rythme pouvait être accéléré, comme dans les années 1986 et 1987, ralenti (430 ha en 1991 et 101 ha en 1992) et, enfin, qu'il pouvait être nul comme ce fut le cas entre 1979 et 1982 où aucun plant ne fut distribué aux villageois. Ces périodes de restriction de l'offre en semences sélectionnées pourraient d'ailleurs avoir favorisé l'adoption du « tout-venant ${ }^{14}$ en secteur villageois (cf. infra : " facteurs économiques »). Ainsi à cette époque, la décision d'adoption dépendait essentiellement des moyens financiers et de la stratégie mise en place par la société d'encadrement.

Mais la privatisation de Palmindustrie, intervenue en 1997, a abouti à une redistribution des rôles entre anciens (planteurs, État) et nouveaux opérateurs (usiniers privés, coopératives de planteurs) de la filière. Face à cette situation nouvelle, la préoccupation principale des planteurs se situe au niveau de leurs approvisionnements en intrants (plants et engrais). Les planteurs se sont donc massivement dirigés vers la station de La Mé pour se procurer du matériel végétal. La décision d'adoption revient dès lors entièrement aux planteurs mais cette liberté d'adopter le palmier à huile dont disposent les planteurs peut être considérée comme une innovation suscitée [17], résultant à la fois de décisions paysannes et du nouvel environnement de la filière. Ce changement se traduit concrètement par une forte croissance du nombre de planteurs et des superficies de palmeraies créées.

Progrès technique et replantation

\section{Replanter en disposant d'un matériel végétal performant}


L'amélioration génétique du palmier à huile a permis de produire un matériel végétal plus performant que celui de la première génération ( $1^{\mathrm{er}} \mathrm{cycle}$ ), avec un rendement à l'hectare et un taux d'extraction supérieurs. Certains planteurs de palmier sélectionné rencontrés lors des enquêtes semblent parfaitement informés sur ce point.

L'adoption de ces nouvelles semences est aussi appréciée par les planteurs qui exploitaient du « tout-venant ". Leurs plantations constituées de palmiers stériles ou produisant des régimes de petites tailles sont, dans le cas des planteurs enquêtés à La Mé, remplacées par des palmiers à haut rendement.

\section{Replantation " palmier après palmier "}

Les difficultés de replantations en palmier observées en Côte d'Ivoire sont les risques de fusariose. Selon Renard et Meunier [18] les dégâts causés par cette maladie cryptogamique peuvent occasionner "des pertes de $50 \%$, voire davantage sur certaines parcelles ". La fusariose se présenterait ainsi comme la contrainte majeure à la replantation. Mais, d'une part, ces risques sont limités à certaines zones de la Côte d'Ivoire seulement et, d'autre part, des résistances génétiques ont été exploitées dans les programmes de sélection et des variétés résistantes à la fusariose sont mises au point et disponibles ${ }^{15}$.

La replantation de palmier après abattage d'une parcelle de palmier ou après jachère pose moins de difficultés que les replantations cacaoyères. Elle n'entraîne guère d'investissements supérieurs en travail et en intrants par rapport à la première plantation. De plus, elle a lieu avec un matériel végétal plus performant.

\section{Facteurs économiques}

L'adoption du palmier par les planteurs répond à un objectif général de la diversification : " générer des revenus d'activités ayant des différences de degrés de risques, de recettes attendues, de liquidité et de saisonnalité » [19].

L'évolution des prix relatifs des matières premières agricoles est en général un facteur déterminant des décisions d'adoption d'une culture. À cet égard, les évolutions du binôme cacao/café en Cote d'Ivoire sont exemplaires, avec un report général du café vers le cacao à partir des années 70 quand le prix du cacao a été rehaussé [20]. Les prix relatifs ont-ils eu la même fonction dans le cas du palmier en Côte d'Ivoire?

\section{Les prix}

Les prix d'achat du cacao et du palmier ont augmenté simultanément et de façon régulière jusqu'en 1987 (figure 7). La période de fort accroissement des superficies villageoises de palmier dans les années 1986-1987 coïncide bien avec une augmentation des prix du palmier (figure 8) mais, d'une part, cette augmentation des prix est régulière depuis 1973 et, d'autre part, la demande est encore à cette période largement suscitée (ou contrainte) par l'offre de Palmindustrie en semences. Il est difficile d'affirmer que ce boom des superficies plantées lors du deuxième plan palmier est lié à une augmentation des prix du palmier, mais c'est un facteur favorable, aux côtés de la diminution de la rente forêt. 
Ensuite, en apparence, la forte diminution des prix du cacao entre 1989 et 1993 ne semble pas avoir provoqué dans ces mêmes années de reports sur la culture du palmier sélectionné. Serait-ce dû au fait que les prix d'achat du régime bord-champ ont également un peu diminué lors de cette même période ? Probablement pas. La diminution des prix des régimes est peu signifiante par rapport à celle du cacao et le palmier à huile a bien dû alors apparaître comme une valeur refuge. La principale raison est que les possibilités de plantations en semences sélectionnées étaient encore fortement liées à l'offre de Palmindustrie. Les planteurs n'avaient donc pas la liberté de répondre aux prix relatifs palmier/cacao plus favorables de l'époque en investissant largement dans le palmier sélectionné.

En revanche, ils avaient la possibilité de répondre aux prix relatifs en investissant largement dans le matériel tout-venant. C'est apparemment ce qui s'est passé. En effet, les données de la figure 8 n'enregistrent que les superficies plantées en matériel sélectionné. Une enquête menée parallèlement par les auteurs dans quatre villages en 1999 indique que les superficies plantées en matériel tout-venant se sont accrues de façon régulière entre 1988 et 1994 (figure 9) alors qu'on a pu noter, sur cette même période, un déclin des superficies plantées en matériel sélectionné, tant sur cet échantillon (figure 10) que de façon globale en Côte d'Ivoire (figures 3 et 4). Cette adoption massive du tout-venant répond donc à une non-disponibilité de semences sélectionnées attribuées par la Palmindustrie à la fin du deuxième plan palmier (en particulier dans les régions proches d'Abidjan) $^{16}$, mais correspond aussi à la chute des cours du cacao entre 1988 et 1993 (le prix passant de 400 à $200 \mathrm{FCFA} / \mathrm{kg}$, voire souvent $150 \mathrm{FCFA} / \mathrm{kg}$ ). Les planteurs semblent donc avoir répondu au déclin des prix du cacao et à la relative stabilité des prix du palmier, d'abord avec du tout-venant quand le matériel végétal sélectionné n'était plus disponible, puis à nouveau avec du matériel sélectionné quand la libéralisation de l'approvisionnement en semences a réactivé l'offre de matériel sélectionné ${ }^{17}$.

Le second boom de croissance en palmier sélectionné observé depuis 1995 est parallèle à une forte augmentation des prix du palmier, passant de 12,5 FCFA/kg en 1993 à 37,50 en 1999.

II sera intéressant de suivre l'évolution de la vente des semences entre 2000 et 2002 à la suite de la chute des cours enregistrée depuis fin 1999, amenant aujourd'hui le prix du kilo de régime à 23 FCFA : la forte diminution du prix d'achat du régime depuis 1 an va-t-elle provoquer une baisse des superficies plantées en palmier ? Probablement pas, du moins pas au profit du cacao et du café car leurs prix ont également rechuté lourdement et on reste donc dans des prix relatifs comparables.

Enfin, le prix peut être un déterminant de l'adoption du palmier non seulement en raison de son niveau, mais aussi de sa régularité. En effet, le prix d'achat du régime de palme est, depuis la privatisation, fixé semestriellement. Cet état de fait est perçu par les planteurs comme un avantage en termes de stabilité de revenu, contrairement au café et au cacao dont les prix varient désormais au jour le jour depuis la campagne 1999/2000. Cela dit, un nouveau mécanisme de fixation du prix du régime de palme est actuellement à l'étude et pourrait remettre en cause cette stabilité relative du prix.

\section{Régularité des revenus}


Sur les exploitations, les régimes de palmier sont récoltés puis acheminés à l'usine deux fois par mois, tout au long de l'année. Les planteurs sont donc rémunérés mensuellement au prorata de leur production. Ils sont quasiment en position de "salariés ". Les planteurs attachent beaucoup d'importance à cette particularité du palmier (qui existe aussi avec l'hévéa).

Cette régularité des revenus du palmier a eu un effet incitatif auprès des salariés de la ville, à tel point que ces derniers ont décidé d'investir dans le palmier pour mieux arrondir les fins de mois difficiles ou préparer leur retraite imminente. Certains salariés sont même des planteurs sans terres, c'est-à-dire qu'ils exploitent des terres qui leur sont cédées pour une période couvrant la durée économique d'une palmeraie. Un accord est établi entre le paysan, propriétaire terrien sans ressources financières, et le salarié ayant des capitaux financiers. Le salarié prend en charge tous les coûts de création et d'entretien de la palmeraie jusqu'à l'entrée en production. Les deux tiers de la palmeraie sont alors exploités par le salarié et le tiers restant revient au paysan. Ce système est appelé " domitchê » par les paysans d'Aboisso. D'autres salariés de la ville qui possèdent des terres (achetées ou cédées) se sont aussi intéressés au palmier à huile, notamment les cadres, et on a vu cidessus que cette catégorie de planteurs pourrait être en forte croissance.

Pour le paysan, le fait de disposer d'un revenu mensuel permet de faire face aux problèmes quotidiens (soins médicaux, déplacements, voyage, participation financière aux cérémonies funéraires). C'est aussi pour lui un facteur de solvabilité et de crédibilité pour avoir accès au crédit notamment auprès des banques ou des instituts d'épargne et de crédits.

\section{Nombreux débouchés et diversité des sources de revenus}

Parmi toutes les cultures pérennes exploitées en Côte d'Ivoire, le palmier à huile est l'une de celles qui présentent le plus de débouchés pour les planteurs.

\section{Les usines de premières transformations}

Ces usines sont les premiers destinataires des régimes villageois. La proximité d'une usine est une source de motivation non négligeable lors de l'adoption de palmier. Avant la privatisation, tout planteur ayant bénéficié des services de la Palmindustrie était obligé de livrer entièrement sa production. Depuis la privatisation, le lieu de vente des régimes dépend (de facto) du planteur. Néanmoins, ces usines restent pour le paysan un client sûr, bien organisé (collecte et date de collecte) et bon payeur, lui accordant encore certains services (encadrement technique, collecte et à un degré moindre fourniture d'intrants à crédit).

\section{Les transformations artisanales en huile}

Ces transformations sont effectuées par quelques planteurs. Mais, la plus grande partie est constituée de femmes qui le font manuellement ou en utilisant des presses mécaniques.

Les planteurs ont recours à ce type de procédé lorsqu'ils ont un retard dans la collecte de leurs régimes par l'EAI, ou s'ils sont confrontés à un problème de liquidités urgent. L'avantage principal que retire le planteur est le paiement au comptant de ces régimes. La vente se fait par tas à raison de trois gros régimes ou quatre petits régimes à 1000 FCFA. Cette transaction est parfois moins 
rémunératrice que la précédente, mais aura permis au planteur d'éviter une perte totale de sa récolte ou de faire face à un problème financier urgent.

\section{La réalisation du capital lors de l'abattage de la palmeraie}

Par rapport à d'autres cultures pérennes en fin du cycle économique, le palmier, en Afrique de l'Ouest, se distingue par un atout particulier. L'abattage d'une palmeraie, quelles qu'en soient les raisons, est une occasion pour le planteur de réaliser un capital. Le palmier abattu permet l'extraction du vin de palme (bangui) dont la consommation est très prisée tant en campagne que dans les agglomérations urbaines. Ce bangui peut ensuite être distillé artisanalement pour obtenir une liqueur (koutoukou) aisément commercialisée.

L'exploitation d'un hectare de palmeraie abattue (140 arbres par ha) pour l'extraction de bangui ou de koutoukou rapporte au planteur une somme comprise entre 350000 FCFA et 420000 FCFA, souvent sous forme de contrat de location, à raison de 2500 FCFA ou 3000 FCFA par arbre. Cet argent peut servir à financer la replantation ou d'autres investissements (habitation, commerce, etc.) et/ou à effectuer les dépenses domestiques.

Les enquêtes font clairement ressortir que les planteurs prennent cette caractéristique en considération au moment où ils adoptent le palmier. Cette possibilité de réaliser un capital lors de l'abattage de la palmeraie est anticipée dès la plantation.

\section{En résumé}

L'adoption du palmier par les planteurs est influencée par plusieurs déterminants lesquels ont suivi un certain processus historique.

* Des décisions politiques initiales de développement du palmier, sous forme de projets qui ont joué un rôle vital dans l'apport d'information et de capital.

* Un développement historique particulier : dans la société civile ivoirienne post-indépendance, les cadres de l'État ont pu bénéficier en priorité de l'information et du capital des projets et de l'accès aux forêts pour l'adoption du palmier.

* Le déterminisme " écologique et historique du déroulement du cycle cacao/café " : paradoxalement, l'adoption du palmier par les paysans correspond plutôt au processus opposé, celui des premières difficultés de replanter le cacao et dans une moindre mesure le café dans un contexte de déforestation.

* Les prix et les déterminants économiques : les prix relatifs jouent un rôle complémentaire puisque la palmier a permis simultanément une augmentation, une diversification et une régularité (mensualisation) des revenus. À cela s'ajoute l'originalité du palmier en Afrique qui est la possibilité de réaliser un capital.

* Des déterminants sociaux : le retour des jeunes dans les villages dans les années 90 a indéniablement contribué à relancer les investissements en palmier. 
* Retour aux facteurs politiques en 1995 : en dépit des contraintes sur le capital, la libéralisation du secteur palmier et le libre accès aux semences sélectionnées sont plutôt favorables à l'adoption du palmier sélectionné dans la mesure où les projets et les structures du passé ont joué un rôle de diffusion de l'information de cette nouvelle culture significatif. La question aujourd'hui est de définir les institutions qui peuvent et qui auraient pour mandat de prendre le relais dans cette diffusion de l'information.

\section{Notes}

${ }^{1}$ Il ne s'agit pas en tant que telle d'une nouvelle culture dans la mesure où des palmeraies naturelles existaient déjà auparavant. Cela dit, la culture de palmier à huile sélectionné peut être considérée comme une nouvelle culture dans la mesure où la conduite des itinéraires techniques, les rendements, les usages et les investissements sont totalement différents entre ces deux types de palmier.

${ }^{4}$ La privatisation de Palmindustrie prend effet en janvier 1997, mais l'annonce préalable de cette privatisation a eu pour effet de modifier l'organisation de la filière quelques années avant. Notamment l'accès aux semences à la station de La Mé est libéralisé dès 1995.

${ }^{5}$ Les plantations industrielles devaient servir de modèle pour les plantations villageoises et assurer un minimum de rentabilité pour le fonctionnement des usines, indispensables au traitement des régimes de palme (qui doivent être usinés dans les 24 à 48 heures).

${ }^{6}$ Les extensions en blocs industriels autour des usines n'étaient plus possibles et il fallait commencer à financer la replantation de ces blocs. Le développement des plantations villageoises devait permettre de compléter l'approvisionnement des usines souvent en état de surcapacité d'usinage.

${ }^{7}$ La demande de création par les producteurs villageois était bien supérieure à l'offre que pouvait réaliser Palmindustrie.

${ }^{8}$ Ce taux reste à prendre avec prudence car on ne connaît pas le taux de conversion réel des graines germées en nombre de plants effectivement plantés.

${ }^{9}$ Nous verrons dans la deuxième partie que notre enquête réalisée à La Mé en 1999 suggère aussi une forte augmentation de l'adoption de palmier (figure 5).

${ }^{10}$ Soixante pour cent des personnes interrogées sont les chefs d'exploitation eux-mêmes, $21 \%$ sont des représentants du chef d'exploitation, et $19 \%$ sont représentants de plusieurs planteurs en même temps, qui ont ainsi regroupé leurs commandes (de 2 à 10 planteurs).

${ }^{11}$ Le coût des graines germées est de 32000 FCFA/ha.

${ }^{12}$ En faisant abstraction des quelques cas de replantations (principalement des cas de remplacement de plantations de palmiers " tout-venant " après que les planteurs en ont vérifié les résultats décevants) mais ces cas restent peu fréquents. Selon une récente analyse réalisée sur la vente de graines germées à La Mé (sur 700 planteurs, soit 500000 graines germées), le taux de replantation après abattage de palmeraies n'est que de 2,2 \% (Durand-Gasselin, 2000, comm. pers. sur la répartition des surfaces créées en extension ou en replantation par région). L'essentiel de l'adoption 
est bien en extension, en création de nouvelles palmeraies.

${ }^{13}$ Sur le graphique en figure 5 : reportée en 2000, date de plantation effective (après 9 mois de pépinière ou 12 mois de pré-pépinière puis pépinière).

${ }^{14}$ Semences vendues à bas prix et non issues de la sélection variétale d'hybrides certifiés et obtenus en station de recherche (CNRA). II s'agit le plus souvent de descendances de semences sélectionnées, dont la productivité est réduite.

${ }^{15}$ Le coût est de 40000 FCFA pour l'équivalent d'un hectare en graines germées.

${ }^{17}$ Ce résultat doit encore être considéré à titre d'hypothèse mais mériterait le même type d'analyse sur un plus large échantillon.

\section{CONCLUSION}

L'information (sur la qualité du matériel végétal notamment), le capital et la disponibilité des intrants sont apparus comme des facteurs essentiels à l'appropriation du palmier sélectionné en milieu villageois. Notamment pour les planteurs villageois, l'adoption du palmier a répondu à un besoin de replantation après vieille caféière et cacaoyère ou après jachère à Chromolaena odorata et à un besoin de financement pour reconstruire un capital plantation.

Le financement à crédit des plantations villageoises entre 1967 et 1990 a permis cet apport de capital. Le relais est pris aujourd'hui par des investissements privés, mais on note que, sur la population enquêtée à La Mé, les cadres sont sur-représentés par rapport à la population actuelle de planteurs de palmier.

La non-disponibilité en semences sélectionnées dans certains villages lors du deuxième plan a favorisé l'usage des semences " tout-venant " par les planteurs. C'est déjà le signe que l'information "palmier " avait pris souche en milieu villageois au point de développer des investissements hors projet. Puis la disponibilité en semences sélectionnées à partir des années 1995, conjuguée à une augmentation des prix bord champs au producteur, a contribué à provoquer une phase d'accroissement des superficies villageoises. Peu d'acteurs et d'experts s'y attendaient. Il en résulte une dynamique des plantations de palmier beaucoup plus forte que les statistiques des projets ne le laissaient penser.

Ce qui reste aujourd'hui difficilement mesurable, c'est la qualité et la nature de l'information dont disposent les planteurs, dans un contexte où l'encadrement technique est nettement réduit. Or ces données vont être déterminantes sur les quantités et sur la qualité de la production de régimes de palme dans les années à venir.

\section{Remerciement}

Les auteurs tiennent à remercier Christian Brunin, Claude Jannot et Tristan Durand-Gasselin pour leurs riches commentaires. De même ils remercient le Directeur de la Station de Recherche de La Mé 
(CNRA), Anatole Koutou, ainsi que Roger Kouamé et Tristan Durand-Gasselin de la station de La Mé, pour leur accueil, leur collaboration scientifique lors des enquêtes menées à La Mé, et leur apport de connaissances agronomiques et variétales de la culture.

\section{REFERENCES}

1. PILLET-SCHWARTZ AM (1978). Les grandes entreprises de culture et de promotion des paysans en Côte d'Ivoire. Études rurales, 70.

2. LOSCH B (1983). L'hévéaculture villageoise en Côte d'Ivoire. Contribution à l'étude de l'évolution des systèmes de production en zone forestière. Université de Montpellier I, Faculté de droit et de sciences économiques; $120 \mathrm{p}$.

3. KOUADIO T (1984). Impact socioéconomique des changements structurels dans les plantations : le cas de la Côte d'Ivoire. CIRES, Abidjan ; 41 p.

4. COLIN JP (1990). La dynamique des systèmes productifs agricoles en basse Côte d'Ivoire. In : Dynamique des systèmes agraires. La dimension économique. Paris : ORSTOM et CNRS : 165-90.

5. MANSO M (1991). Conflits fonciers et enjeux économiques. Le cas des planteurs d'Elaeis guineensis en basse Côte d'Ivoire. Cahiers Ivoiriens de Recherche Économiques et Sociales, 1 : 39-66.

6. HIRSCH R (1996). Les filières palmier à huile et hévéa dans la zone franc. Enquête sur la relance des économies de la zone franc après la dévaluation. Ministère de la Coopération, CFD ; $124 \mathrm{p}$.

7. RUF F (1992). Après la forêt, quelle stabilisation de l'agriculture de plantation ? Le cas du département d'Abengourou, Côte d'Ivoire. Eschborn et Montpellier : GTZ \& CIRAD, CIRAD-SAR, 1992/04; $72 \mathrm{p}$.

8. RUF F (1987). Éléments pour une théorie sur l'agriculture des régions tropicales humides. I. De la forêt, rente différentielle au cacaoyer, capital travail. Agronomie Tropicale 42 : 218-32.

9. JANNOT C (1999). Le fonds d'extension et de replantation pour la culture du palmier à huile (FER) en Côte d'Ivoire. Note provisoire. Montpellier ; Cirad-Tera : 53 p.

10. COLIN JP (1990). La mutation d'une économie de plantation en basse Côte d'Ivoire. Paris : ORSTOM, coll. À travers champs ; $284 \mathrm{p}$.

11. LEONARD E (1997). La reproduction de la société agraire dans la région de Sassandra. Intensification ou décapitalisation ? In : GUILLAUME A, IBO J, N'GUESSAN K, eds. Croissance démographique, développement agricole et environnement à Sassandra (Sud-Ouest de la Côte d'Ivoire). ORSTOM, ENSEA, GIDIS : 137-60.

12. MOLLARD E (1992). Fronts pionniers, économie de plantation paysanne et intensification en basse Côte d'Ivoire. In : CHAUVEAU JP, et al. eds. L'innovation en milieu rural : synthèse des groupes de travail de la table ronde du LEA et textes des contributions au séminaire LEA, Session 1991-1992. Montpellier : ORSTOM.

13. RUF F (1995). De l'économie familiale de plantation à l'économie familiale de replantation cacao en Côte d'Ivoire et Indonésie. Actes du séminaire. Fertilité du milieu et stratégies paysannes sous les 
tropiques humides. 13-17 novembre 1995, Montpellier : CIRAD, ministère de la Coopération : 451-63.

14. OSWALD M (1997). Recomposition d'une société au travers de plusieurs crises : la société rurale Bété. Thèse de $3^{\mathrm{e}}$ cycle. Institut national économique Paris-Grignon. Paris.

15. RUF F (1999). Comment et pourquoi la Côte d'Ivoire produit durablement plus d'un million de tonnes de cacao ? Afrique Agriculture, $268: 21-5$.

16. COLIN JP (1990). La dynamique des systèmes productifs agricoles en basse Côte d'Ivoire. In : Dynamique des systèmes agraires. La dimension économique. Paris : ORSTOM et CNRS : 165-90.

17 LOSCH B, VARLET F (1995). Quelles innovations institutionnelles face à l'ajustement structurel ? Le cas de la restructuration des filières café et cacao au Cameroun et en Côte d'Ivoire. Actes du $14^{\mathrm{e}}$ séminaire d'économie rurale Innovation et société. Quelles agricultures ? Quelles innovations ? Volume II : Les diversités de l'innovation, 13-16 septembre 1993, Montpellier : CIRAD, ORSTOM, INRA : 123-32.

18. RENARD JL, MEUNIER J (1983). Research for durable resistance to vascular wilt disease (Fusarium oxysporum $f$. sp. elaeidis) of oil palm (Elaeis guineensis). In : LAMBERTI F, WALLER JM, VAN DER GRAAFF NA, eds. Durable resistance in crops. Plenum Publishing Corporation : 287-90.

19. DELGADO C, SIAMWALLA A (1999). Rural economy and farm income diversification in developping countries. In : Food Security, diversification and ressource management : refocusing the role of agriculture ? : 126-43.

20. RUF F (1995). Booms et crises du cacao. Les vertiges de l'or brun. Paris : Karthala ; $459 \mathrm{p}$.

21. JANNOT (1999). Opération d'appui au développement. Étude des bassins de collecte des huileries de palme en Côte d'Ivoire (texte provisoire), CIRAD-TERA ; 6 p. 


\section{Illustrations}

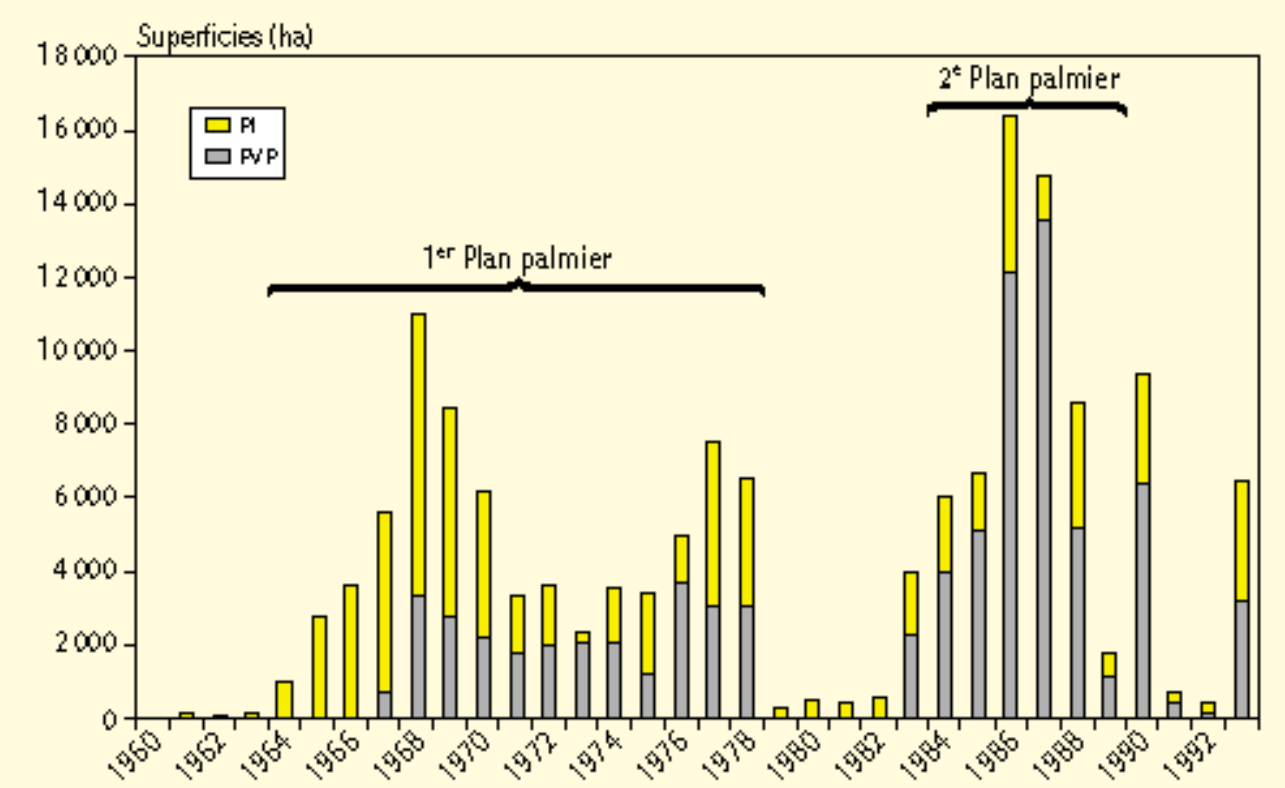

Figure 1. Superficies plantées entre 1960 et 1993 : plantations industrielles (PI) et plantations villageoises (PV) (d'après Hirsch [6]).

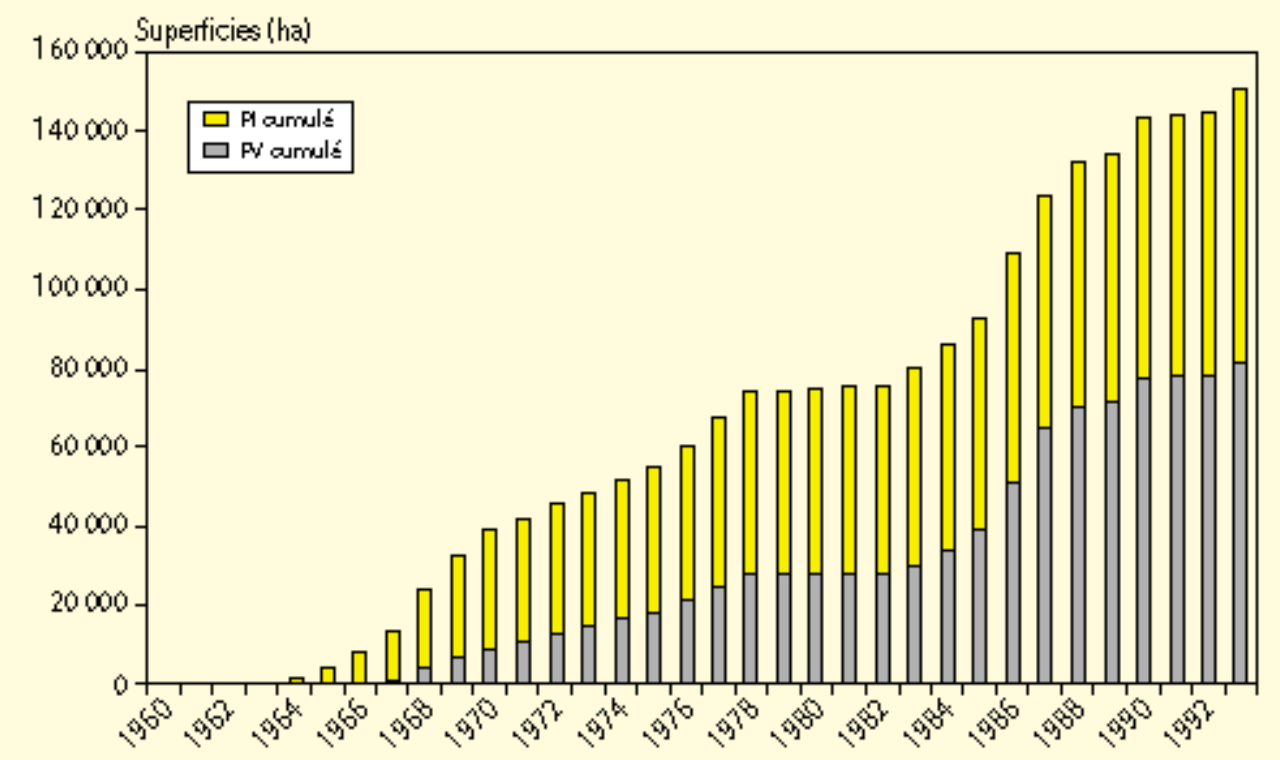


Figure 2. Superficies plantées cumulées entre 1960 et 1993 (villageoises : PV et industrielles : PI) (d'après Hirsch [6]).

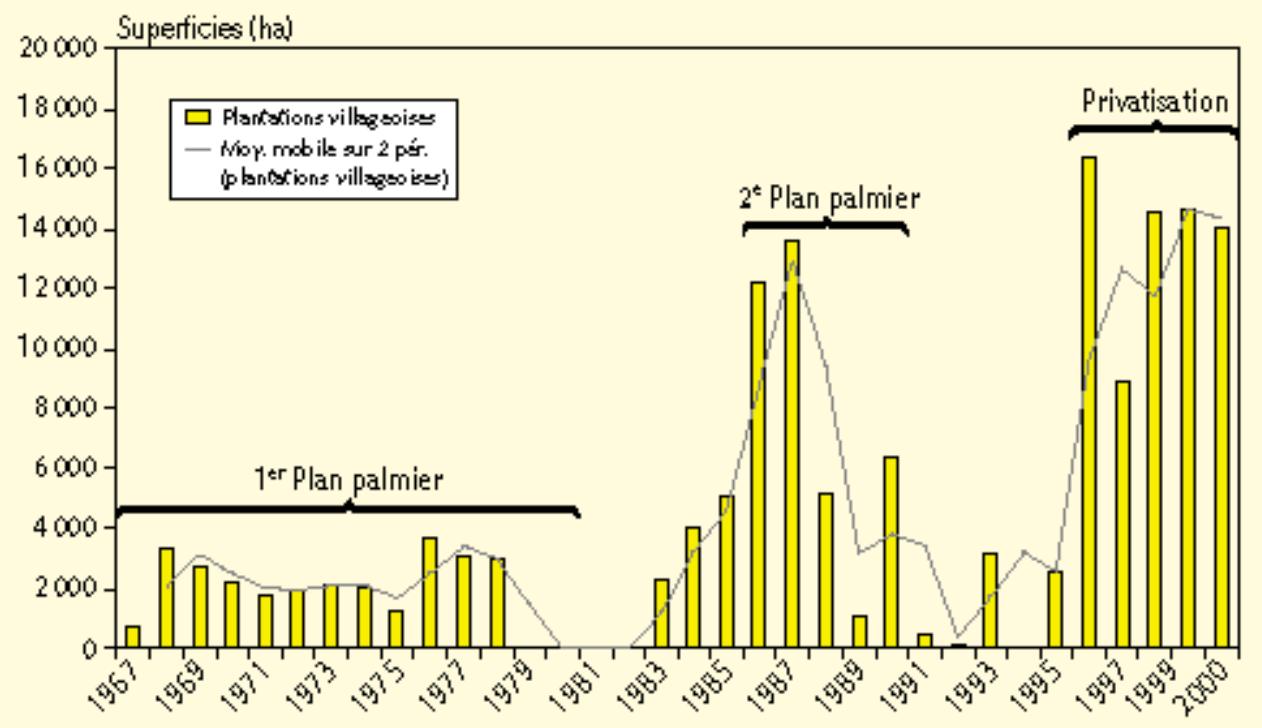

Figure 3. Superficies plantées en secteur villageois entre 1967 et 2000 (d'après Hirsch [6], Jannot [comm. pers.] et CNRA La Mé [année 2000, estimations]).

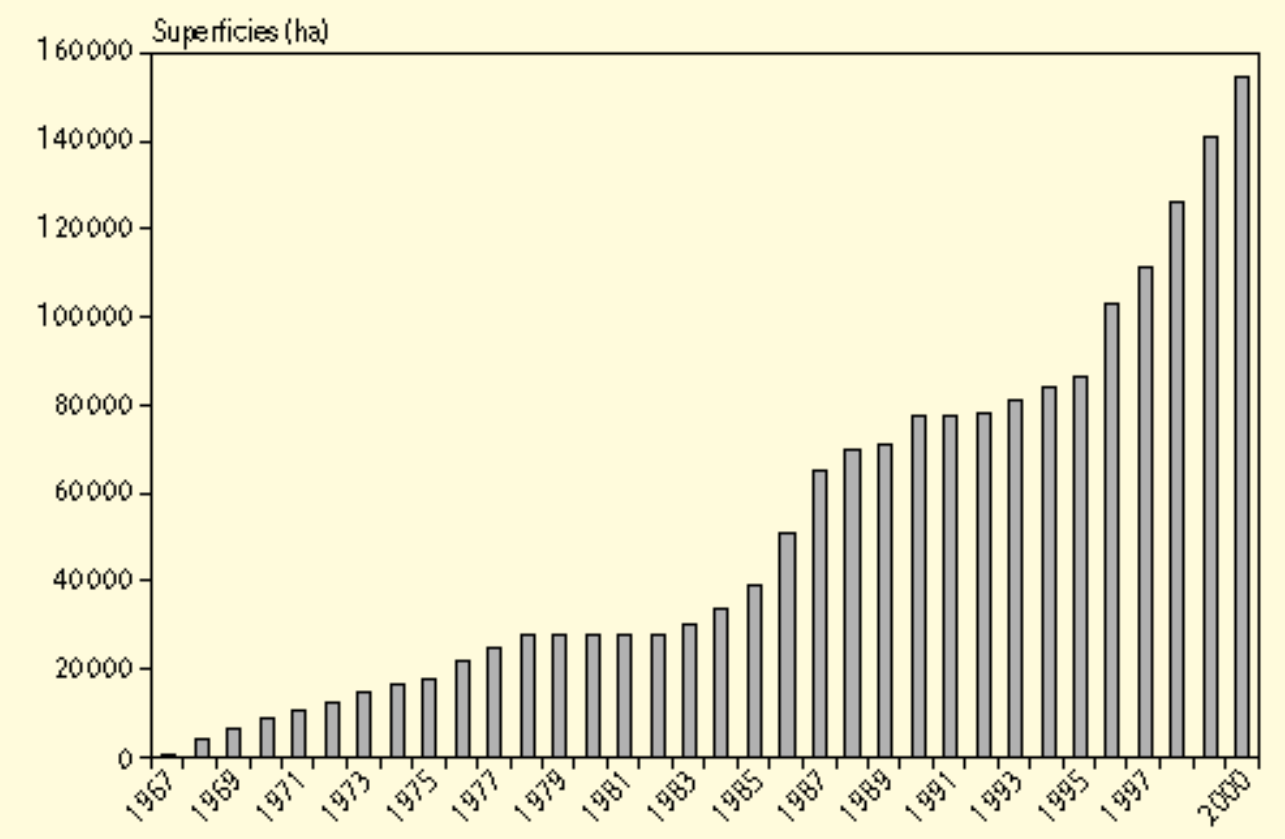


Figure 4. Superficies plantées cumulées en secteur villageois entre 1967 et 2000

(d'après Hirsch [6], Jeannot [comm. pers.] et CNRA La Mé [année 2000, estimations]).

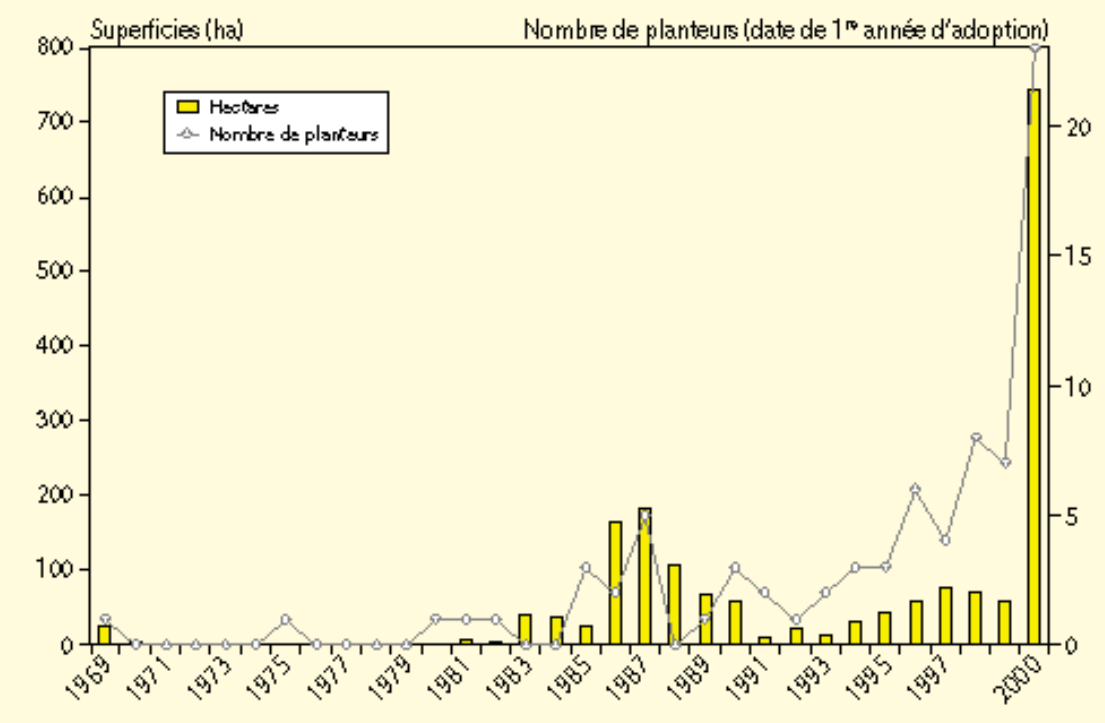

Figure 5. Superficies plantées et date de première adoption (enquête à La Mé sur 80 planteurs).

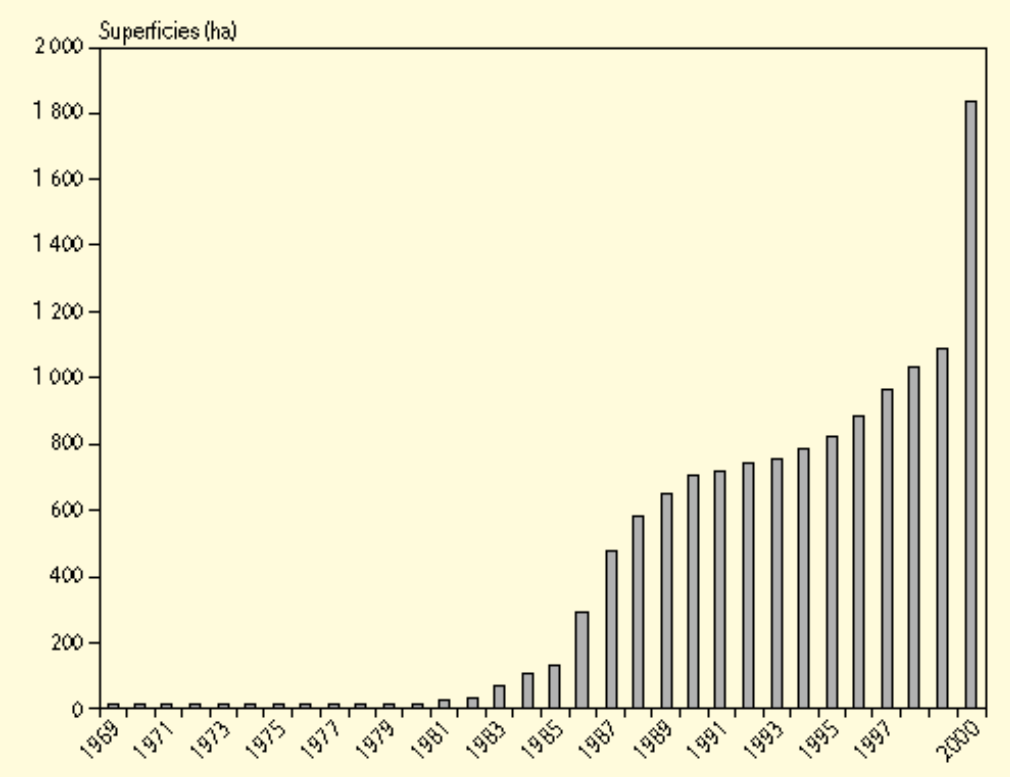

Figure 6. Superficies plantées cumulées (enquête à La Mé sur 80 planteurs) Les superficies plantées en 2000 correspondent aux achats de graines germées en 1999 (données recueillies lors de l'enquête de La Mé en juin-juillet 1999). Les graines 
germées ne sont plantées qu'après 9 mois de pépinières (ou 12 mois de pré-pépinière et pépinière), donc en 2000 pour les achats enregistrés en 1999.

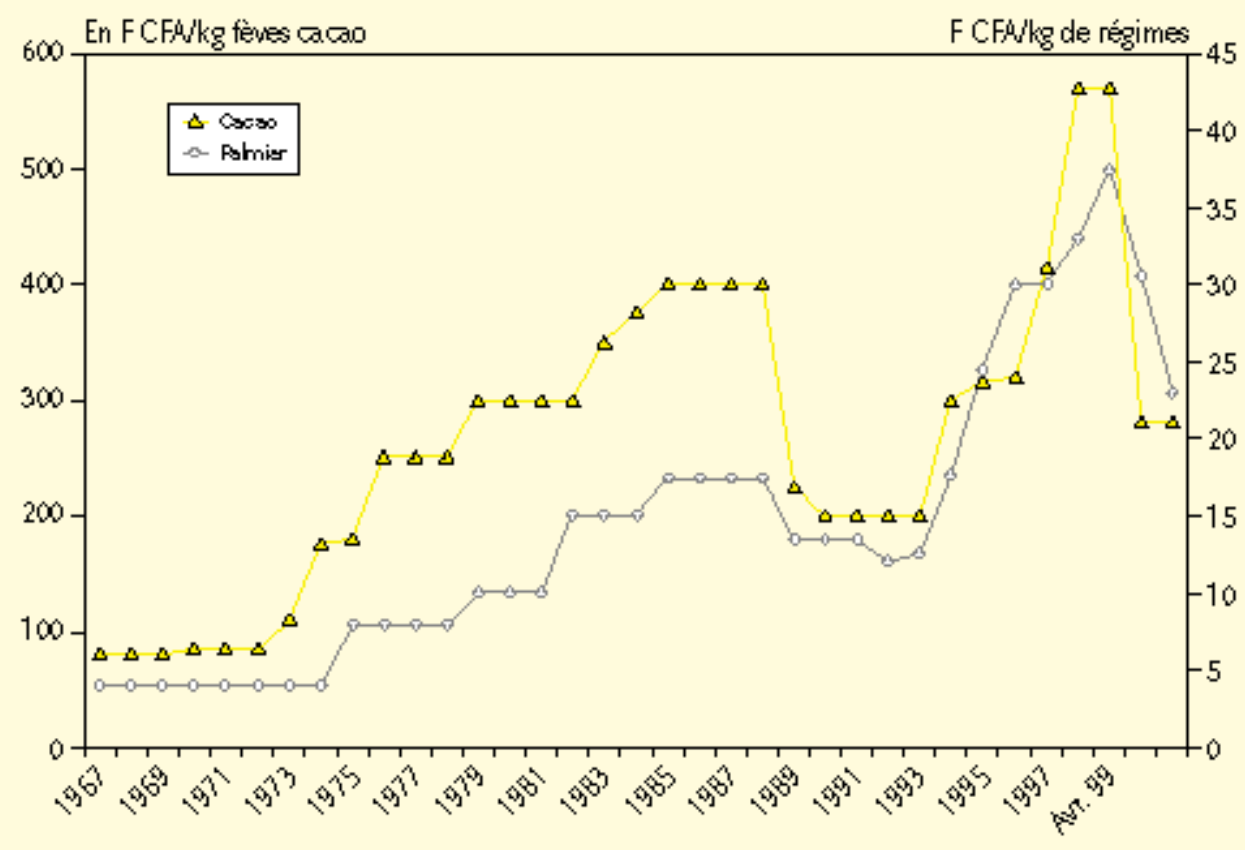

Figure 7. Évolution des prix d'achat au producteur du cacao et du palmier.

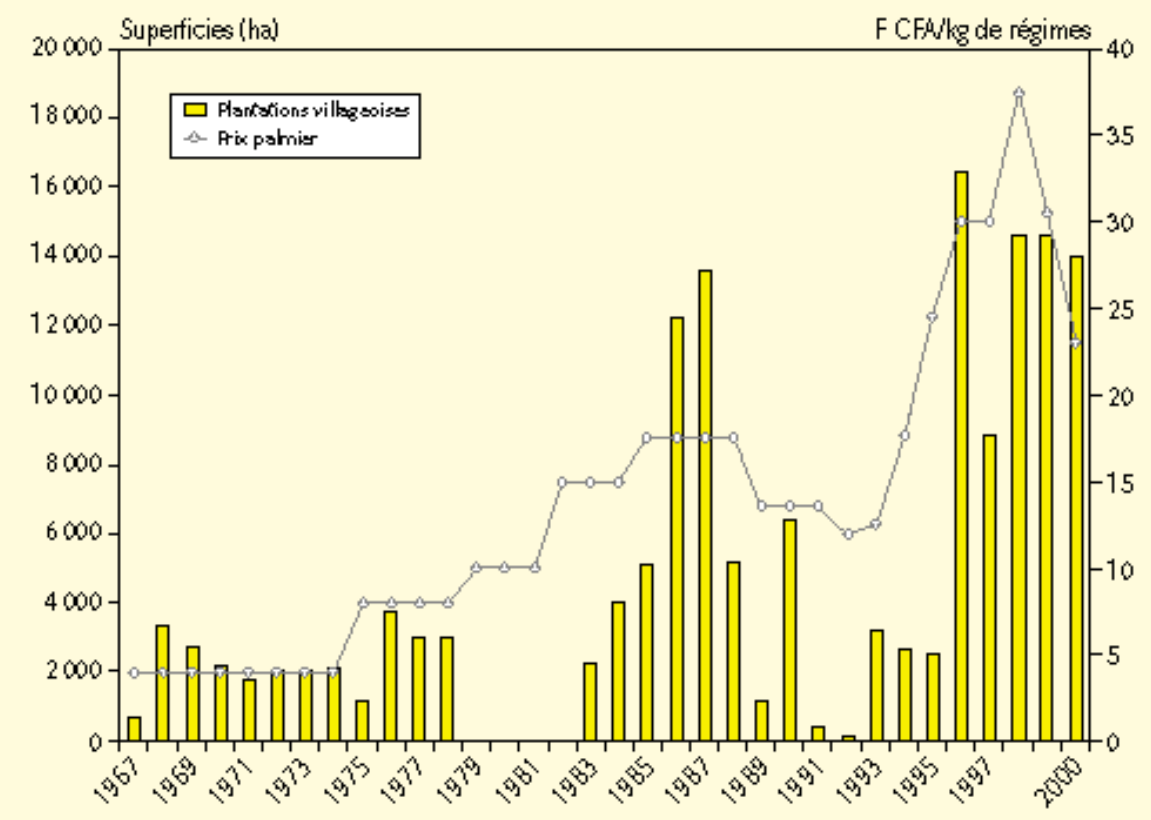


Figure 8. Superficies plantées en secteur villageois entre 1967 et 2000 et évolution du prix d'achat au producteur.

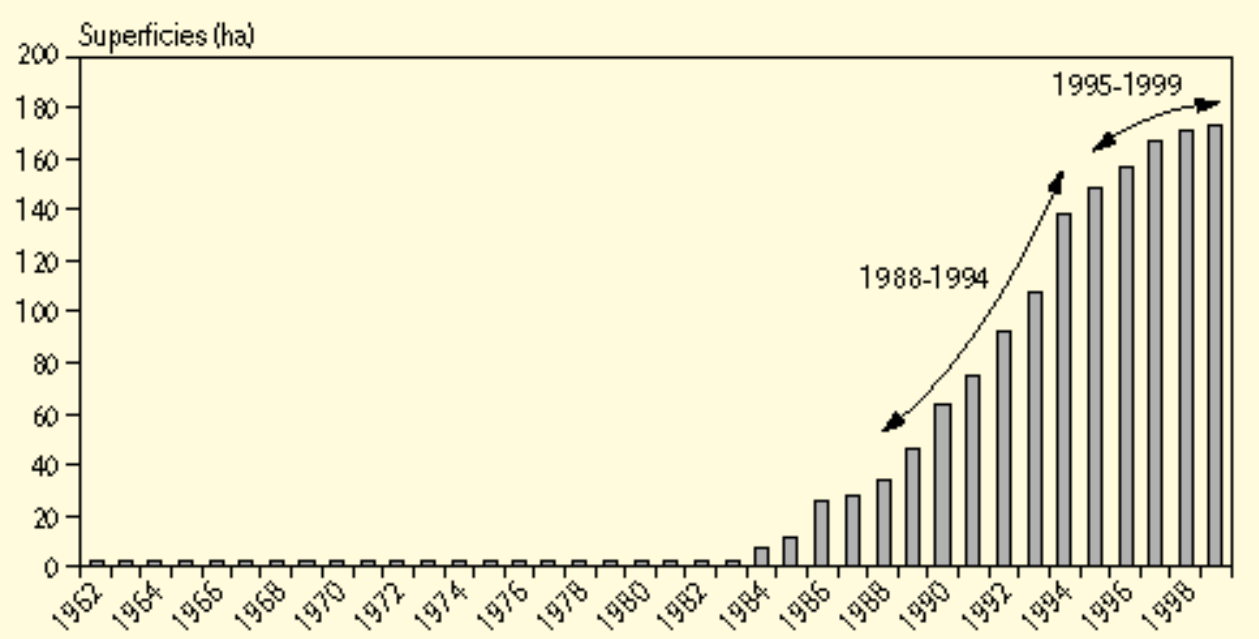

Figure 9. Superficies cumulées de palmier « tout-venant » (enquête sur 38 planteurs).

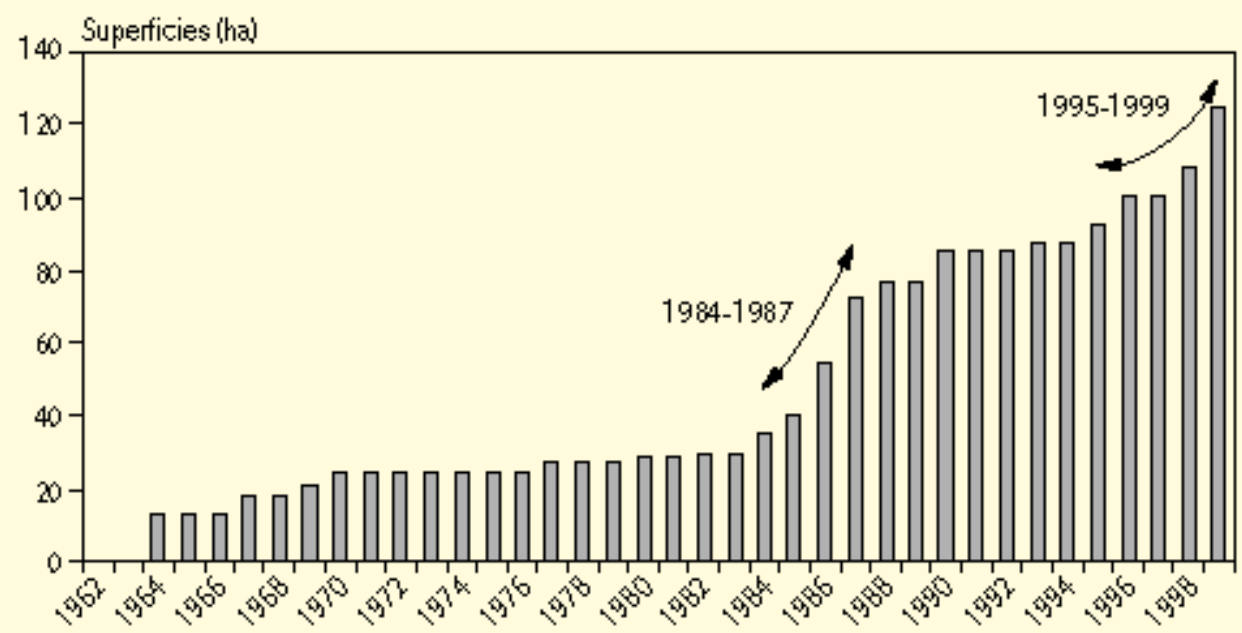

Figure 10. Superficies cumulées de palmier selectionné (enquête sur 38 planteurs). Source figures 9 et 10 : Nos enquêtes en 1999 dans 4 villages (1 village dans la région de Dabou, 3 villages dans le sud-est). $\mathrm{N}=38$ planteurs (parmi l'échantillon total) qui ont déclaré avoir planté du « tout venant ». Parmi ces 38 planteurs, 28 sont planteurs du village de Yassap. 


\begin{tabular}{|c|c|c|c|c|}
\hline Date & Phase correspondante & $\begin{array}{c}\text { Financement } \\
\text { extérieur }\end{array}$ & $\begin{array}{l}\text { Orga- } \\
\text { nisme }\end{array}$ & $\begin{array}{l}\text { Coût global } \\
\text { du projet } \\
\text { (inclut les } \\
\text { fin ancements } \\
\text { propres) }\end{array}$ \\
\hline 1963 & Conception du Premier Plan palmier & $\begin{array}{l}\text { 1,15 milliard FCFA } \\
\text { (aide non remboursable) }\end{array}$ & CEE & \\
\hline 1965 & Création de plantationsen usufruits & 8,1 milliards FCFA & FED & \\
\hline 1967 & $\begin{array}{l}\text { Création de PV, } \\
\text { abandon du système d'usufruit }\end{array}$ & 7,7 millions FF & CCCE & \\
\hline 1969 & $\begin{array}{l}\text { Création de Palmindustrie } \\
\text { et Palmivoire (Soc. Économie mixte) }\end{array}$ & 17,1 millions de $\$$ US & $\begin{array}{l}\text { BIRD, } \\
\text { CCCE, }\end{array}$ & \\
\hline 1971 & Deuxième projet BIRD & 7 millions de $\$$ US & BIRD & \\
\hline 1974 & Troisième projet BIRD & $?$ & BIRD & \\
\hline \multirow[t]{3}{*}{1977} & Palmind ustrie devient Société d'État. & & & \\
\hline & - Projet Sud-Ouest & 4,9 milliards FCFA & BIRD & $\begin{array}{l}11,3 \text { milliards } \\
\text { FCFA }\end{array}$ \\
\hline & - Projet Sassandra & 2,5 millions de $f$ & $\mathrm{CDC}$ & - \\
\hline $1979-1982$ & Arrêt des plantations & & & \\
\hline $1983-1985$ & Progra mme $5^{\circ} \mathrm{FED}$ & 10 millions écus & FED & \\
\hline $1985-1990$ & Deuxième Plan Palmier & 33 millia rds FCFA & $\begin{array}{c}\text { BIRD, } \\
\text { CDC, } \\
\text { CCCE, } \\
\text { BEl, FED }\end{array}$ & 90 milliards FCFA \\
\hline
\end{tabular}

Tableau 1. Financements des ensembles agro-industriels de palmier à huile ( $P V, P I$, huileries, $P M E A)^{a}$.

\begin{tabular}{|c|c|c|c|c|c|c|}
\hline \multirow{3}{*}{$\begin{array}{l}\text { 10 Plan } \\
\text { 1963-1982 }\end{array}$} & \multicolumn{2}{|c|}{ Sup totales } & \multicolumn{2}{|c|}{ Sup industrielles } & \multicolumn{2}{|c|}{ Sup villageoises } \\
\hline & 75681 & $100 \%$ & 47943 & $63 \%$ & 27737 & $37 \%$ \\
\hline & $60 \%$ & & $72 \%$ & & $35 \%$ & \\
\hline \multirow{2}{*}{$\begin{array}{l}2^{\circ} \mathrm{Plan}+5^{\circ} \mathrm{FED} \\
1983-1990\end{array}$} & 67529 & $100 \%$ & 17752 & $26 \%$ & 49777 & $74 \%$ \\
\hline & $40 \%$ & & $28 \%$ & & $65 \%$ & \\
\hline \multirow{2}{*}{$\begin{array}{l}\text { Total } \\
\text { 1963-1982 }\end{array}$} & 143210 & $100 \%$ & 65695 & $46 \%$ & 77515 & $54 \%$ \\
\hline & $100 \%$ & & $100 \%$ & & $100 \%$ & \\
\hline
\end{tabular}

Tableau 2. Superficies plantées entre 1963 et 1990 (hectares) 


\begin{tabular}{|lrrr}
\hline & Total & \multicolumn{1}{c}{ PV } & \multicolumn{1}{c}{ PI } \\
\hline 1963-1982:1* Plan & 3290 & 1734 & 2084 \\
$1985-1990: 2^{\star}$ Plan & 9590 & 7250 & 2340 \\
$1995-2000:$ Privatisation & ND & 11828 & ND \\
\hline
\end{tabular}

Tableau 3. Superficies annuelles moyennes plantées lors des trois phases (hectares)

Source : période 1963-1990 : Hirsch ; période 1995-2000 : CNRA, La Mé.

\begin{tabular}{|c|c|c|c|c|}
\hline & $\begin{array}{c}\text { Effectif } \\
\text { de la classe }\end{array}$ & $\begin{array}{c}\text { Total } \\
\text { superficies } \\
\text { par classe }\end{array}$ & $\begin{array}{l}\text { Superficie } \\
\text { moyen ne par } \\
\text { exploitation }\end{array}$ & $\begin{array}{c}\text { Man cuvres } \\
\text { par } \\
\text { exploitation }\end{array}$ \\
\hline Gadre retraité & 4 & 269 & 67,2 & 19,0 \\
\hline $\begin{array}{l}\text { Cadre moyen et supérieur } \\
\text { en activité }\end{array}$ & 14 & 380 & 29,3 & 10,8 \\
\hline Gadre subalteme, instituteur, etc. & 18 & 57 & 3,3 & 0,9 \\
\hline $\begin{array}{l}\text { Planteur paysan } * 1 \% \text {, ayant plus de } 50 \% \\
\text { de son exploitation en palmier }\end{array}$ & 18 & 189 & 10,2 & 5,0 \\
\hline $\begin{array}{l}\text { Planteur paysan } * 2 * \text {, ayant moins de } 50 \% \\
\text { de son exploitation en palmier }\end{array}$ & 23 & 63 & 2,8 & 1,8 \\
\hline
\end{tabular}

Tableau 4. Superficies en palmier et nombre de manœuvres selon le type d'exploitation, avant 1999

Quelques cas particuliers difficiles à regrouper en catégories professionnelles ne sont pas mentionnés.

\begin{tabular}{|lcc}
\hline & $\begin{array}{c}\text { Total } \\
\text { des semences ach etées }\end{array}$ & $\begin{array}{c}\text { Moyenne } \\
\text { par exploitation }\end{array}$ \\
\hline Cadre retraité & 355 & 89 \\
Cadre moyen et supérieur en activité & 179 & 13 \\
Cadre subalteme, instituteur, etc. & 60 & 3,3 \\
Planteur paysan * 1 \%, ayant plus de $50 \%$ & 37 & 2,4 \\
de son exploitation en palmier & & 3,1 \\
Planteur paysan * 2 \%, ayant moins de $50 \%$ & 72 & \\
de son exploitation en palmier & & \\
\hline
\end{tabular}

Tableau 5. Achats de semences en 1999 exprimés en équivalents hectares de palmiers plantés en 2000 


\begin{tabular}{|lccccc}
\hline & $\begin{array}{c}\text { Revenu } \\
\text { palmier } \\
\text { (+ complt) }\end{array}$ & $\begin{array}{c}\text { Revenu } \\
\text { cacao } \\
\text { café et } \\
\text { autre culture }\end{array}$ & $\begin{array}{c}\text { Salaire } \\
\text { Aide }\end{array}$ & $\begin{array}{c}\text { Total } \\
\text { familiale }\end{array}$ \\
\hline Cadre retraité & 4 & 0 & 0 & 0 & 4 \\
Cadre moyen et supérieur en activité & 2 & 0 & 9 & 0 & 11 \\
Cadre subalteme, instituteur, etc. & 0 & 1 & 14 & 0 & 15 \\
Planteur paysan * $1 \%$, ayant plus de $50 \%$ & 12 & 2 & 0 & 1 & 15 \\
de son exploitation en palmier & 4 & 11 & 0 & 3 & 18 \\
Planteur paysan * $2 \%$, ayant moins de $50 \%$ & & 14 & 23 & 4 & 63 \\
de son exploitation en palmier & 22 & 14 & & & \\
Total & & & & & \\
\hline
\end{tabular}

Tableau 6. Origine du capital selon le type d'exploitant (effectif planteurs)

\begin{tabular}{|lr|cccc} 
& Forêt & Jachère & $\begin{array}{r}\text { Vieux } \\
\text { cacao }\end{array}$ & $\begin{array}{c}\text { Vieux } \\
\text { café }\end{array}$ & Palmier \\
\hline Cadre retraité & 269 & 0 & 0 & 0 & 0 \\
Cadre moyen et supérieur en activité & 188 & 5 & 10 & 18 & 12 \\
Cadre subalteme, instituteur, etc. & 12 & 11,5 & 2 & 12,5 & 4 \\
$\begin{array}{l}\text { Planteur paysan * 1 \%, ayant plus de 50\% } \\
\text { de son exploitation en palmier }\end{array}$ & 27 & 84 & 7 & 45 & 2 \\
$\begin{array}{l}\text { Planteur paysan * 2 } \% \text {, ayant moins de 50\% } \\
\text { de son exploitation en palmier }\end{array}$ & 5 & 35 & 4 & 4 & 12 \\
Total & 501 & 135,5 & 23 & 79,5 & 30 \\
\hline
\end{tabular}

Tableau 7. Superficies en palmier (ha) par type d'exploitant et précédent cultura 